\title{
Robust 3D Head Tracking and Its Applications
}

\author{
Wooju Ryu and Daijin Kim \\ Intelligent Multimedia Laboratory, Dept. of Computer Science and Engineering, \\ Pohang University of Science and Technology (POSTECH), Pohang, Korea \\ $\{$ wjryu, dkim\}@postech.ac.kr
}

\begin{abstract}
The head tracking is a challenging work and a useful application in the field of computer vision. This paper proposes a fast 3D head tracking method that is working robustly under a variety of difficult conditions. First, we obtain the pose robustness by using the 3D cylindrical head model (CHM) and dynamic template. Second, we also obtain the robustness about the fast head movement by using the dynamic template. Third, we obtain the illumination robustness by modeling the illumination basis vectors and by adding them to the previous input image to adapt the current input image. Experimental results show that the proposed head tracking method outperforms the other tracking method using the fixed and dynamic template in terms of the small pose error and the higher successful tracking rate and it tracks the head successfully even if the head moves fast under the rapidly changing poses and illuminations in a speed of 10-15 frames/sec. The proposed head tracking method has a versatile applications such as a head gesture TV remote controller for the handicapped people and a drawing tool by the head movement for the entertainment.
\end{abstract}

\section{Introduction}

For the 3D head tracking, many researchers have used simple geometric head models such as a cylinder [1], 2], an ellipsoid [3], or a head-like 3D shape [4] to recover the global head motion. They assume that the shape of the head model does not change during tracking, which means that it does not have the shape parameters. The global head motion can be represented by a rigid motion, which can be parameterized by 6 parameters; three for 3D rotation and three for 3D translation. Therefore, the number of the model parameters is only 6 . Among three different geometric 3D head models, we take the cylindrical head model due to the robustness to the pose variation and the general applicability and the simplicity. It is more appropriate to approximate the 3D shape of the generic faces than the ellipsoid model. Also, it requires a small number of parameters and its fitting performance is less sensitive to their initialization than the head-like 3D shape model.

To be more robust about the extreme pose and fast head movement, dynamic template technique has been proposed [2]. Although the dynamic template technique can treat the extreme head movement, it has a problem that can fail the head tracking due to the accumulated fitting error. To remedy this problem, [2] 
proposed the re-registration technique that stored the reference frames and used them when the fitting error became large. The dynamic template may cover the gradually changing illumination because the template is updated every frames. However, it can not cover all kinds of illumination changes, specifically for the rapidly changing illumination. 1] removed the illumination effects by adding the illumination basis vectors to the template. This approach can cover the rapidly changing illumination.

We derive a novel full-motion recovery under perspective projection that combines the dynamic template and re-registration technique 2 and the removal of illumination effect by adding the illumination basis vectors [1. Also, we update the reference frames according to the illumination condition of input image. This approach provides a new head tracking method which is robust to the extreme head poses, the fast head movement, the rapidly changing illumination.

\section{Full Motion Recovery Under the Rapidly Changing Illuminations}

\subsection{Full Motion Recovery}

The cylinder head model assumes that the head is shaped as a cylinder and the face is approximated by the cylinder surface. The 3D cylinder surface represented as $\mathbf{x}=\left[\begin{array}{lll}x & y & z\end{array}\right]^{\mathrm{T}}$ and the 2D image pixel coordinate is represented as $\mathbf{u}=\left[\begin{array}{ll}u & v\end{array}\right]^{\mathrm{T}}$. If we take the perspective projection function, the $2 \mathrm{D}$ image pixel coordinate $\mathbf{u}$ is given by

$$
\mathbf{u}=P(\mathbf{x})=\frac{f_{L}}{z}\left[\begin{array}{ll}
x & y
\end{array}\right]^{\mathrm{T}},
$$

where $f_{L}$ is the focal length.

When the cylinder surface point $\mathbf{x}$ is transformed by the rigid motion vector $\mathbf{p}$, the rigid transformation function $M(\mathbf{x} ; \mathbf{p})$ of $\mathbf{x}$ can be represented by

$$
M(\mathbf{x} ; \mathbf{p})=R \mathbf{x}+T,
$$

where $R \in \mathbb{R}^{3 \times 3}$ and $T \in \mathbb{R}^{3 \times 1}$ are the $3 \mathrm{D}$ rotation matrix and the $3 \mathrm{D}$ translation vector, respectively. We take the twist representation [8, whose detailed derivation is given in 9]. According to the twist representation, the 3D rigid motion model $M(\mathbf{x} ; \mathbf{p})$ is given by

$$
M(\mathbf{x} ; \mathbf{p})=\left[\begin{array}{ccc}
1 & -w_{z} & w_{y} \\
w_{z} & 1 & -w_{x} \\
-w_{y} & w_{x} & 1
\end{array}\right]\left[\begin{array}{l}
x \\
y \\
z
\end{array}\right]+\left[\begin{array}{l}
t_{x} \\
t_{y} \\
t_{z}
\end{array}\right]
$$

where $\mathbf{p}=\left[\begin{array}{llllll}w_{x} & w_{y} & w_{z} & t_{x} & t_{y} & t_{z}\end{array}\right]^{\mathrm{T}}$ is the $3 \mathrm{D}$ full head motion parameter vector. The warping function $W(\mathbf{x} ; \mathbf{p})$ in Eq. (??) is completely defined by using $P(\mathbf{x})$ and $M(\mathbf{x} ; \mathbf{p})$ as

$$
\begin{aligned}
W(\mathbf{x} ; \mathbf{p}) & =P(M(\mathbf{x} ; \mathbf{p})) \\
& =\left[\begin{array}{l}
x-y w_{z}+z w_{y}+t_{x} \\
x w_{z}+y-z w_{x}+t_{y}
\end{array}\right] \frac{f_{L}}{-x w_{y}+y w_{x}+z+t_{z}}
\end{aligned}
$$


When we consider the illumination basis vectors, the objective function for the robust $3 \mathrm{D}$ head tracking is given by

$$
\text { minimize } \sum_{\mathbf{x}}\left[I(W(\mathbf{x} ; \mathbf{p}), t)-\sum_{i=1}^{b_{N}}\left(q_{i}+\Delta q_{i}\right) \mathbf{b}_{i}(\mathbf{x})-I(W(\mathbf{x} ; \mathbf{p}+\Delta \mathbf{p}), t+1)\right]^{2},
$$

where $\mathbf{p}$ and $q_{i}$ are the $3 \mathrm{D}$ rigid motion parameter and the $i-t h$ illumination coefficient, respectively, and $\Delta \mathbf{p}$ and $\Delta q_{i}$ are the updated parameters computed by solving optimization problem.

\subsection{Linear Approximation}

To solve the Eq. (6), we need to approximate the nonlinear equation to the linear equation about the $\Delta \mathbf{p}$ and $\Delta \mathbf{q}$ as

$$
\begin{aligned}
& I(W(\mathbf{x} ; \mathbf{p}), t)-\sum_{i=1}^{b_{N}}\left(q_{i}+\Delta q_{i}\right) \mathbf{b}_{i}(\mathbf{x})-I(W(\mathbf{x} ; \mathbf{p}+\Delta \mathbf{p}), t+1) \\
\approx & I(W(\mathbf{x} ; \mathbf{p}), t)-I(W(\mathbf{x} ; \mathbf{p}), t+1) \\
& -\sum_{i=1}^{b_{N}} q_{i} \mathbf{b}_{i}(\mathbf{x})-\sum_{i=1}^{b_{N}} \Delta q_{i} \mathbf{b}_{i}(\mathbf{x})-\nabla \mathbf{I} \frac{\partial \mathbf{W}}{\partial \mathbf{p}} \Delta \mathbf{p} .
\end{aligned}
$$

Let us define the error image, the steepest descent image, and the Hessian matrix $H$ as

$$
\begin{aligned}
& E(\mathbf{x})=I(W(\mathbf{x} ; \mathbf{p}), t)-I(W(\mathbf{x} ; \mathbf{p}), t+1)-\sum_{i=1}^{b_{N}} q_{i} \mathbf{b}_{i}(\mathbf{x}) \\
& S D(\mathbf{x})=\left[\nabla \mathbf{I} \frac{\partial \mathbf{W}}{\partial p_{1}}, \ldots, \nabla \mathbf{I} \frac{\partial \mathbf{W}}{\partial p_{6}}, \mathbf{b}_{1}(\mathbf{x}), \ldots, \mathbf{b}_{b_{N}}(\mathbf{x})\right] \\
& H=\sum_{\mathbf{x}} S D(\mathbf{x})^{\mathrm{T}} S D(\mathbf{x}) .
\end{aligned}
$$

Then, the model parameters $[\Delta \mathbf{p} \quad \Delta \mathbf{q}]^{\mathrm{T}}$ can be obtained as

$$
\left[\begin{array}{c}
\Delta \mathbf{p} \\
\Delta \mathbf{q}
\end{array}\right]=H^{-1} \sum_{\mathbf{x}} S D(\mathbf{x})^{\mathrm{T}} E(\mathbf{x})
$$

\subsection{Parameter Update}

At every frame, we iteratively update the parameters $\Delta \mathbf{p}$ and $\Delta \mathbf{q}$ simultaneously. Before the iterative process, we need to set the previous input image patch as the current template and set the initial parameters $\mathbf{p}=\mathbf{p}_{0}$ and $\mathbf{q}=0$, where $\mathbf{p}_{0}$ is the previous motion parameters. At every iteration, we compute the new error image and the steepest descent image. When computing the new error image, the parameter $\mathbf{p}$ in the first term $I(W(\mathbf{x} ; \mathbf{p}), t)$ should be kept as $\mathbf{p}_{0}$ because it is used as the template image. Table 1 summarizes the overall process of the iterative parameter update of $\mathbf{p}$ and $\mathbf{q}$, where $\epsilon_{1}$ and $\epsilon_{2}$ are the threshold values. 
Table 1. Overall process of the parameter update

(1) Set the previous input image patch as template image.

(2) Initialize the parameters as $\mathbf{p}=\mathbf{p}_{0}$ and $\mathbf{q}=0$.

(3) Compute $E(\mathbf{x})$ and $S D(\mathbf{x})$.

(4) Compute the Hessian matrix $H$.

(5) Compute the incremental parameter $\Delta \mathbf{p}$ and $\Delta \mathbf{q}$.

(6) Update the parameter by $\mathbf{p} \leftarrow \mathbf{p}+\Delta \mathbf{p}$ and $\mathbf{q} \leftarrow \mathbf{q}+\Delta \mathbf{q}$.

(7) If $\Delta \mathbf{p}<\left|\epsilon_{1}\right|$ and $\Delta \mathbf{q}<\left|\epsilon_{2}\right|$ then stop. Otherwise goto (3).

\section{Robust 3D Head Tracking}

The dynamic template and re-registration method firstly suggested in 2 for robust head tracking. In this chapter we will review the dynamic template and explain how to generate illumination basis vectors and the modified re-registration algorithm under the rapidly changing illumination.

\subsection{Dynamic Template}

We briefly review the dynamic template method for the robust head tracking [2]. Since the fixed template can not cover the all kinds of head motions, we consider the dynamic template to obtain the long-term robustness of head motion. The cylindrical model can not represent the head shape exactly, the template from the initial frame can not cover the current input image when the head pose is extremely changed. The dynamic template method assumes that the previous input image patch is the current template image.

\subsection{Illumination Basis Vectors}

Although the dynamic template approach can cover the gradually changing illumination, it can not cover the rapidly changing illumination effectively. To tackle the rapidly changing illumination, we propose to use the linear model. To build illumination model, we generate the head images whose illuminations are changed in five different directions ( left, right, up, down, and front side ), collect illumination images, and apply the principle component analysis (PCA) to the collected images after subtracting the mean image.

\subsection{Re-registration}

We describe what frames are referenced and how to execute the re-registration process with the illumination vectors are considered. While tracking the head motion, the fitting error can be accumulated. Therefore, when the fitting error is over a certain threshold value, we need to re-register to prevent the accumulation error. In the early step of tracking, before accumulation error is over the threshold value, we record the input image $I$ and its motion parameter $\mathbf{p}$ 
as the reference frames (reference DB). The reference frames are classified with the head pose $w_{x}, w_{y}, w_{z}$ and each class in the reference DB is represented by one reference frame. When the re-registration is executed, the reference frame which corresponds to the current head pose is selected from the reference DB. If the illumination is changed, we can not use the old reference DB because the current input image has different illumination condition with the reference frame. Therefore we update the reference DB when the norm of the illumination parameter $\mathbf{q}$ is larger than a threshold value, after the re-registration is performed.

\section{Experimental Results}

We developed the illumination-robust 3D head tracking system using the realtime algorithm explained above. We used the desktop PC(Pentium IV $3.2 \mathrm{GHz}$ ) and Logitech Web Camera. The average tracking speed is about the 10 frames per second when the illumination basis vectors are used and is about 15 frames per second when they are not used. To initialize automatically, we used the face and eye detector based on MCT+Adaboost 10, and AAM face tracker to find face boundary and face feature points.

\subsection{Extreme Pose and Fast Head Movement}

In this experiments, we compared the 3D head tracking using the fixed and dynamic template.

First, we captured the test sequence with extreme head motion about the tilting and yawing. Fig. 1 (a) shows the result of the 3D head tracking, where each row corresponds to the 3D head tracking using the fixed template and the 3D head tracking using the dynamic template, respectively. As you see, the 3D head tracking using the fixed template starts to fail from the 48 - th frame and loses the head tracking completely at the $73-t h$ frame. After the $73-t h$ frame, the tracking can not be performed any more. However, we can get a stable head tracking throughout the entire frames using the dynamic template. Fig. 1 1 (b) compared the measured 3D head poses (tilting, yawing, rolling) when the poses changed much, where the left and right column correspond to the fixed template and the dynamic template, respectively, and the ground truth poses are denoted as the dotted line.

Second, we evaluated our head tracking system when the head move very fast. The test sequence has 90 frames with one tilting and two yawings. Fig. 2. (a) shows the result of 3D head tracking using the test image sequence. While the tracking using the fixed template is failed at frame 31, the tracking using the dynamic template is succeeded throughout the whole test sequence. Fig. 2f(b) compared the measured 3D head poses when the head moves fast.

As you see in the above experiments, the fixed template produces the large measurement error in the 3D head poses, but the dynamic template produces the very accurate $3 \mathrm{D}$ head pose measurements. 


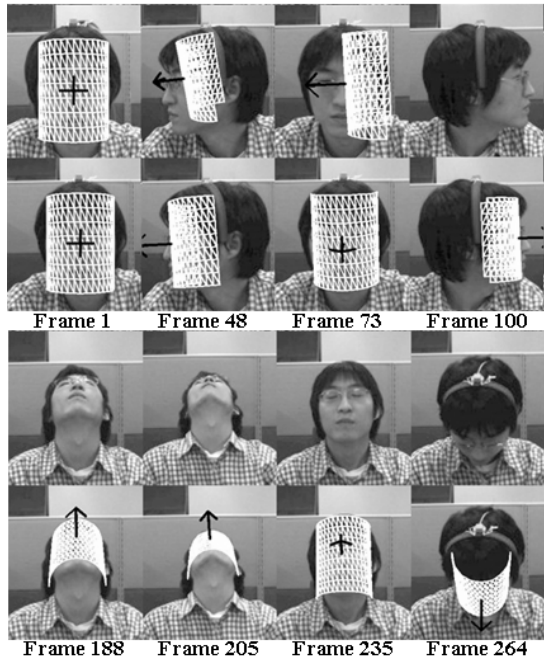

(a)
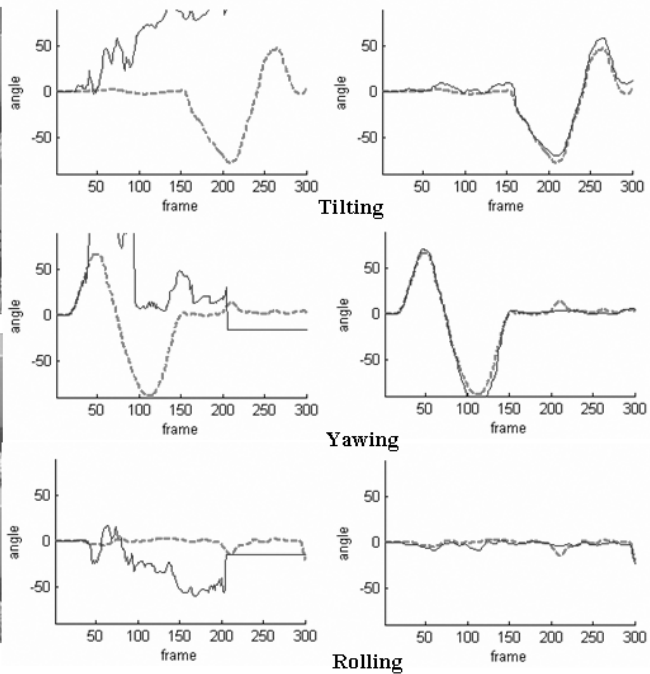

(b)

Fig. 1. Comparison of the head tracking results and the measured 3D head poses in the case of changing poses

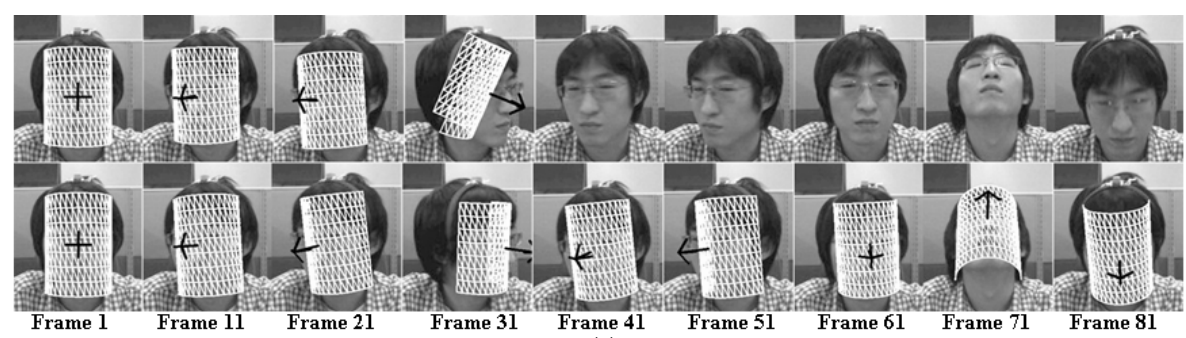

(a)
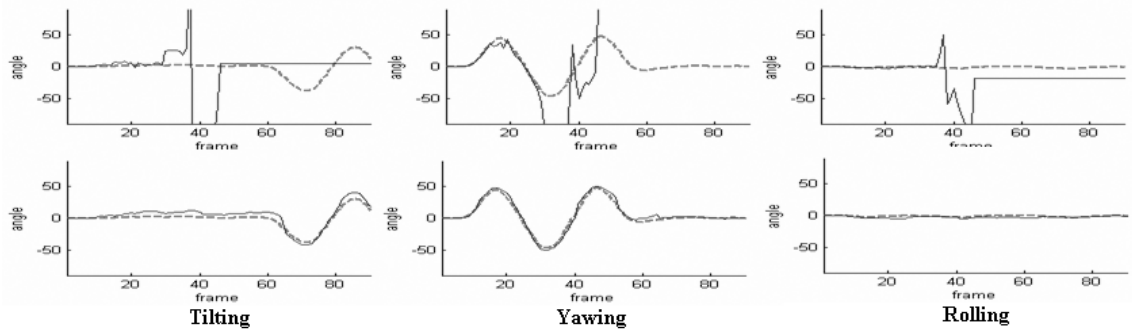

(b)

Fig. 2. Comparison of head tracking results and the measured $3 \mathrm{D}$ head poses in the case of the fast head movement

\subsection{Rapidly Changing Illumination}

We tested how the proposed head tracking system is executed when the illumination condition is changed rapidly. The test sequence has three rapidly changing 


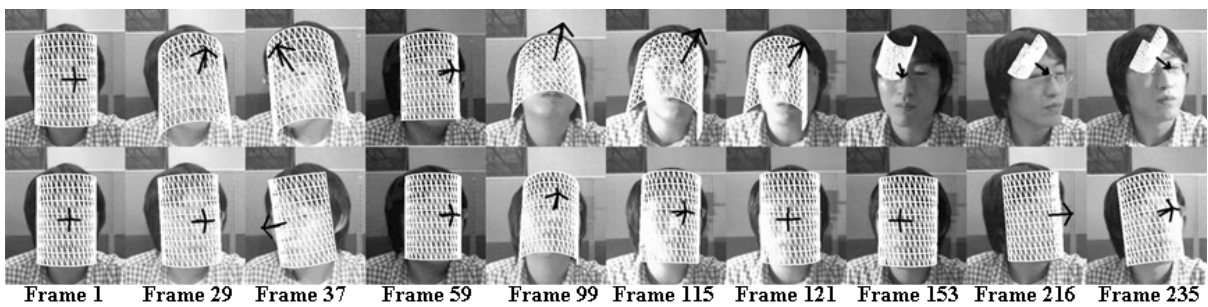

Fig. 3. Comparison of the tracking results under the rapidly changing illumination

illuminations (front, left, and right). Fig. 3 compares the head tracking results, where each row corresponds to the head tracking result with the dynamic template only and the head tracking result with the dynamic template using the illumination basis vectors. As you see, when we use the dynamic template only, it shows the very unstable tracking results under the rapidly changing illuminations. On the other hand, when we use the dynamic template with the illumination basis vectors, the tracking results are very stable throughout the entire frames even though the illumination condition changes rapidly.

\subsection{Rapidly Changing Pose, Head Movement and Illumination}

To test the proposed head tracking methods more faithful, we built the head moving database1 of the 15 different peoples which includes five different illuminations (left, right, up, down, and front). The ground truth of the head rotation is measured by the 3D object tracker (Fastrak system). Table 4.3 summarizes the head tracking experiments on the IMH DB, where the number in each test sequence denotes the number of frames, $r_{\text {track }}$ denotes the ratio of the number of successfully tracked frames over the number of total frames, and the average pose error $E_{p}$ is computed by computing the sum of of tilting, yawing, and rolling pose error between the ground truth and the estimated angle every frame and averaging the pose error sums over the entire frames. From this table, we know that the proposed tracking method using the dynamic template with the illumination basis vectors is successfully tracking the whole image sequences in the IMH DB.

\subsection{Application 1: Face Remote Controller}

We developed the face remote controller (FRC) for real world application. The FRC is the remote controller which is controlled by the head gesture instead of the hand. The head gestures are used for moving the current cursor to the left, right, up, and down side, where the cursor is designated to move discretely between buttons. And, the eye blinking is used for generating the button click event. We apply the FRC to a TV remote controller system using the CCD camera that can zoom in/out and is located on the top of the TV. The TV

1 We call this database the IMH DB [1]. 
Table 2. The tracking results on the IMH DB

\begin{tabular}{l|c|c|cc}
\hline & $\begin{array}{c}\text { Fixed } \\
\text { template }\end{array}$ & $\begin{array}{c}\text { Dynamic } \\
\text { template }\end{array}$ & $\begin{array}{c}\text { Dynamic template with } \\
\text { the illumination basis vectors }\end{array}$ \\
\hline \hline Seq. 1 (679) & $r_{\text {track }}=0.05$ & $r_{\text {track }}=0.89$ & $r_{\text {track }}=1$ & $E_{p}=3.89$ \\
\hline Seq. 2 (543) & $r_{\text {track }}=0.1$ & $r_{\text {track }}=0.54$ & $r_{\text {track }}=1$ & $E_{p}=3.34$ \\
\hline Seq. 3 (634) & $r_{\text {track }}=0.06$ & $r_{\text {track }}=0.26$ & $r_{\text {track }}=1$ & $E_{p}=3.75$ \\
\hline Seq. 4 (572) & $r_{\text {track }}=0.07$ & $r_{\text {track }}=0.44$ & $r_{\text {track }}=1$ & $E_{p}=4.81$ \\
\hline Seq. 5 (564) & $r_{\text {track }}=0.06$ & $r_{\text {track }}=0.9$ & $r_{\text {track }}=1$ & $E_{p}=5.75$ \\
\hline Seq. 6 $(663)$ & $r_{\text {track }}=0.08$ & $r_{\text {track }}=0.39$ & $r_{\text {track }}=1$ & $E_{p}=4.41$ \\
\hline Seq. 7 (655) & $r_{\text {track }}=0.07$ & $r_{\text {track }}=0.48$ & $r_{\text {track }}=1$ & $E_{p}=3.89$ \\
\hline Seq. 8 (667) & $r_{\text {track }}=0.05$ & $r_{\text {track }}=0.88$ & $r_{\text {track }}=1$ & $E_{p}=4.29$ \\
\hline Seq. 9 (588) & $r_{\text {track }}=0.09$ & $r_{\text {track }}=0.38$ & $r_{\text {track }}=1$ & $E_{p}=4.49$ \\
\hline Seq. 10 $(673)$ & $r_{\text {track }}=0.23$ & $r_{\text {track }}=0.48$ & $r_{\text {track }}=1$ & $E_{p}=2.92$ \\
\hline Seq. 11(672) & $r_{\text {track }}=0.07$ & $r_{\text {track }}=0.42$ & $r_{\text {track }}=1$ & $E_{p}=5.30$ \\
\hline Seq. 12 $(504)$ & $r_{\text {track }}=0.04$ & $r_{\text {track }}=0.59$ & $r_{\text {track }}=1$ & $E_{p}=8.21$ \\
\hline Seq. 13 (860) & $r_{\text {track }}=0.37$ & $r_{\text {track }}=0.53$ & $r_{\text {track }}=1$ & $E_{p}=5.38$ \\
\hline Seq. 14 (694) & $r_{\text {track }}=0.13$ & $r_{\text {track }}=0.72$ & $r_{\text {track }}=1$ & $E_{p}=2.19$ \\
\hline Seq. 15 (503) & $r_{\text {track }}=0.25$ & $r_{\text {track }}=0.58$ & $r_{\text {track }}=1$ & $E_{p}=3.79$ \\
\hline
\end{tabular}

watcher sits in the chair which is approximately 5 meters far from the TV. Fig. 4 shows how to perform the cursor movement and button click with the head gesture and the eye blinking, and how the FRC is applied to the TV remote controller system.

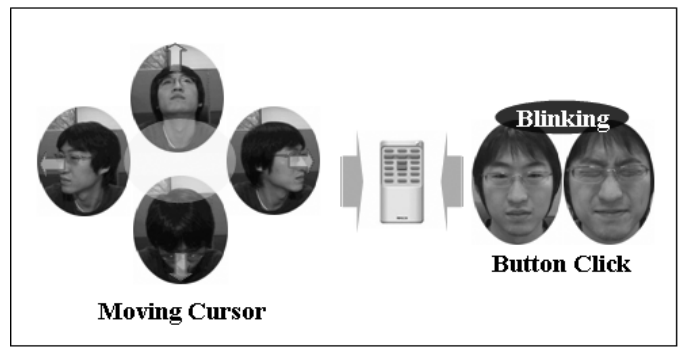

(a)

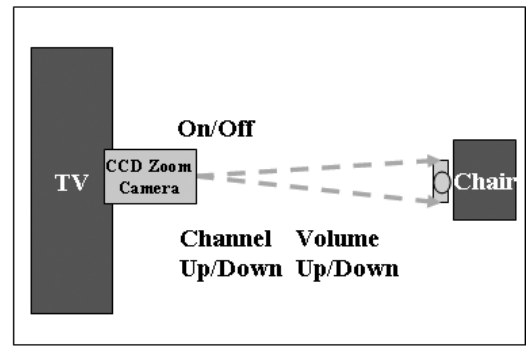

(b)

Fig. 4. Cursor movement and click using the head gesture and the eye blinking

\subsection{Application 2: Drawing Tool by Head Movement}

We also applied the head movement to develop a drawing tool. Basically, we use the head movement instead of the mouse or tablet pen to move the mouse cursor, where the center point on the front cylinder surface is used as the position of the mouse cursor. We define three state as "Wait", "Move", and "Draw" to organize the drawing tool by the head movement (DTHM). When the state is "Wait", the DTHM system does nothing and just wait for the eye blinking. Once 

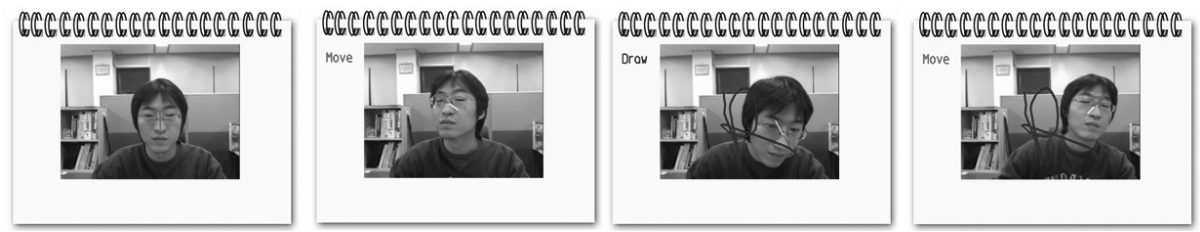

Fig. 5. Drawing tool by head movement

the eye blinking has been occurred in the state of "Wait", the DTHM system changes the state from "Wait" to "Move". In the state of "Move", we can move the mouse cursor where you want to start drawing. If there are no movements in the state of "Move" for some time, the state is changed from "Move" to "Draw", and then we can draw a shape by moving the head. If you want to stop drawing and move mouse cursor, stop head moving and wait for some time until the state is changed from "Draw" to "Move". Fig. 5 shows how to execute the DTHM system. Initially, the system is in the state of "WAIT" and changes to the "Move" state by blinking the eye. Then, the system is changed to the "Draw" state by doing nothing for some time and we draw the "R" character by moving the cursor appropriately. We change the state from the "Draw" to "Move" to move the cursor to the next drawing position.

\section{Conclusion}

We proposed a new framework for the 3D head tracking using the 3D cylinder head model, which combined several techniques such as dynamic template, reregistration, and the removal of illumination effects using the illumination basis vectors.

Also, we proposed the new object function that added the linear illumination model to the existing objective function based on LK image registration and derived the iterative updating formula of the model parameters such as the rigid motion parameter $\mathbf{p}$ and the illumination coefficient vector $\mathbf{q}$ at the same time. We modified the overall process of the existing re-registration technique such that the reference frames could be updated when the illumination condition was changed rapidly.

We performed many intensive experiments of 3D head tracking using the IMH DB. We evaluated the head tracking performance in term of the pose error and the successful tracking rate. The experiment results showed that the proposed head tracking method was the most accurate and stable among other tracking methods using the fixed and dynamic template.

We also developed the face TV remote controller for the handicapped people and the drawing tool by head movement for the entertainment to prove the versatile applicability of the proposed 3D head tracking method. 


\section{Acknowledgement}

This work was financially supported by the Ministry of Education and Human Resources Development(MOE), the Ministry of Commerce, Industry and Energy(MOCIE) and the Ministry of Labor(MOLAB) through the fostering project of the Lab of Excellency. Also, it was partially supported by the Intelligent Robotics Development Program, one of the 21st Century Frontier R\&D Programs funded by the Ministry of Commerce, Industry and Energy of Korea.

\section{References}

1. Cascia, M., Sclaroff, S., Athitsos, V.: Fast, reliable head tracking under varying illumination: An approach based on robust registration of texture-mapped $3 \mathrm{~d}$ models. IEEE Trans. Pattern Analysis and Machine Intelligence(PAMI) 22, 322-336 (2000)

2. Xiao, J., Moriyama, T., Kanade, T., Cohn, J.: Robust full-motion recovery of head by dynamic templates and re-registration techniques. International Journal of Imaging Systems and Technology 13, 85-94 (2003)

3. Basu, S., Essa, I., Pentland, A.: Motion regularization for model-based head tracking. In: Proceedings of the International Conference on Pattern Recognition(ICPR), vol. 3, p. 611 (1996)

4. Malciu, M., Preteux, F.: A robust model-based approach for 3d head tracking in video sequences. In: Proceedings of the Fourth IEEE International Conference on Automatic Face and Gesture Recognition, p. 169. IEEE Computer Society Press, Los Alamitos (2000)

5. Lucas, B., Kanade, T.: An iterative image registration technique with an application to stereo vision. In: Proceedings of the 7th International Joint Conference on Artificial Intelligence (IJCAI), pp. 674-679 (1981)

6. Baker, S., Matthews, I.: Lucas-Kanade 20 Years On: A Unifying Framework: Part 1, tech. report CMU-RI-TR-02-16, Technical Report. Robotics Institute, Carnegie Mellon University (2002)

7. Baker, S., Gross, R., Matthews, I.: Lucas-Kanade 20 Years On: A Unifying Framework: Part 3, tech. report CMU-RI-TR-03-35, Technical Report. Robotics Institute, Carnegie Mellon University (2003)

8. Bregler, C., Malik, J.: Tracking people with twists and exponential maps. In: IEEE Conference on Computer Vision and Pattern Recognition(CVPR), pp. 8-15. IEEE Computer Society Press, Los Alamitos (1998)

9. Murray, R., Li, Z., Sastry, S.: A Mathematical Introduction to Robotic Manipulation. CRC Press, Boca Raton, USA (1994)

10. Froba, B., Ernst, A.: Face detection with the modified census transform. In: Proceedings of the IEEE Conference on Automatic Face and Gesture Recognition, pp. 91-96. IEEE Computer Society Press, Los Alamitos (2004)

11. Ryu, W., Sung, J., Kim, D.: Asian Head Movement Video Under Rapidly Changing Pose, Head Movement and Illumination AHM01, Technical Report. Intelligent Multimedia Lab, Dep. of CSE, POSTECH (2006) 